\title{
The Assessment of Viability of M. Tuberculosis after Exposure to CPC Using Different Methods
}

\author{
Gomathi Sekar, R. Lakshmi, and N. Selvakumar \\ Department of Bacteriology, National Institute for Research in Tuberculosis (ICMR), Chetput, Chennai, \\ Tamil Nadu 600031, India \\ Correspondence should be addressed to Gomathi Sekar; gomathi_sekar@hotmail.com
}

Received 26 June 2013; Revised 25 November 2013; Accepted 11 December 2013; Published 28 January 2014

Academic Editor: Gary Dykes

Copyright (C) 2014 Gomathi Sekar et al. This is an open access article distributed under the Creative Commons Attribution License, which permits unrestricted use, distribution, and reproduction in any medium, provided the original work is properly cited.

Settings. National Institute for Research in Tuberculosis, Chennai. Objective. To assess the proportion of metabolically active cells of Mycobacterium tuberculosis after exposed to CPC using FDA-EB vital staining and viable counts on LJ medium. Mycolic acid content in M. tuberculosis after exposure to CPC was estimated using HPLC. Methods. Clinical isolates of M. tuberculosis and standard reference strain M. tuberculosis H37Rv were used for FDA-EB, viable count, and HPLC. Results. FDA/EB consistently stained 70-90\% of log phase cells as green and the remaining cells as red-orange. After CPC treatment, $65-70 \%$ of the cells stained red-orange. The viability counts were comparable to 0-day controls. Synthesis of mycolic acids in mycobacteria was reduced when exposed to CPC using HPLC due to the decreased metabolic activity of the organisms. Conclusion. The cells are metabolically inactive during storage with CPC but these cells grew well on LJ medium after removal of CPC. The viability of M. tuberculosis was maintained in CPC with minimal reduction. Mycolic acid content was reduced if the cells of M. tuberculosis were treated with CPC for 7 days. All the above findings provide yet another evidence for the damage of cell wall of $M$. tuberculosis.

\section{Introduction}

Tuberculosis caused by Mycobacterium tuberculosis is a public health problem which has increased in importance during the last 12 years, due in part to the increasing number of cases caused by the association of acquired immunodeficiency syndrome (AIDS) and the appearance of multiple drug-resistant strains [1-3]. Other mycobacteria which are often indistinguishable from tuberculosis have also increased. Sputum samples collected from remote areas to central laboratories were transported in CPC. Use of cetylpyridinium chloride (CPC) as transport medium for the recovery of $M$. tuberculosis is established and accepted procedure. CPC plays a dual role as liquefaction as well as decontamination agent. It is usually recommended to allow minimum exposure of sputum specimen with decontamination agents (e.g., $\mathrm{NaOH}$ in modified Petroff's method) as they tend to reduce the viability of the bacilli. But sputum specimens collected with CPC are processed after 5-7 days of collection. The cell wall integrity of $M$. tuberculosis was disturbed during transportation but when the CPC was washed from the sample, the cell wall regained and grew normally on LJ medium (publication in process). Mode of action of CPC on M. tuberculosis and its recovery from sputum is not clearly documented. Moreover, liquid CPC has some usage constraint. Hence the study was carried out to check and document the action of CPC on the viability of M. tuberculosis and the use of powder form of CPC as alternative to liquid form of CPC.

Attempt was made to use powder form of CPC (P-CPC) replacing the use of liquid form of CPC (L-CPC) used at a concentration of $7.6 \mathrm{mg} / \mathrm{mL}$ of sputum. In order to detect the viability of mycobacteria in CPC, collected sputum samples were stored at ambient temperature for up to 7 days and checked for viability at every time point. To assess the viable counts (VC) on LJ medium; the proportion of metabolically active cells of $M$. tuberculosis after exposure to CPC using FDA-EB vital staining method and the mycolic content of $M$. tuberculosis is also done. 


\section{Methods}

Four clinical isolates and standard reference strain $M$. tuberculosis (H37Rv) were used for the study. Log phase cultures were used for the study after subculturing from original LJ slopes. Middlebrook 7H9 broth supplemented with $10 \%$ bovine serum albumin and $0.5 \%$ Tween 80 was prepared.

2.1. Preparation of Single Cell Suspension. The 7-day-old cultures were suspended and vortexed in 7H9 medium. After incubation for 5 days, turbidity of the culture was adjusted to McFarland 4 units and centrifuged (Eppendorf AG, 22231 Hamberg, Germany) at 14,000 rcf for 10 minutes. The pellet was resuspended in $6 \mathrm{~mL}$ of phosphate buffered saline (PBS) buffer and 4 aliquots of $1.5 \mathrm{~mL}$ each were made. The first aliquot (on zero day) was aseptically transferred to a $10 \mathrm{~mL}$ syringe and filtered through a $5 \mu \mathrm{m}$ membrane filter (Sartorius) to obtain single cell suspension.

2.2. FDA-EB Staining. One hundred microliter of single cell suspension was mixed with $100 \mu \mathrm{L}$ of freshly prepared FDAEB staining solution and incubated for $15 \mathrm{~min}$ in dark at room temperature. A wet mount was prepared and sealed to prevent evaporation and observed under fluorescent microscope at a magnification of 450x (Olympus BX 40, Japan) with blue filter. A total of 200 cells were counted in duplicates and differentiated on the basis of colour.

2.3. Viable Count. Viable count of single cell suspension was set up on LJ medium by the usual procedure described above. The second aliquot (7-day-old culture suspension) was left at ambient conditions for 7 days. The third and fourth aliquotes were mixed with powder form of CPC. Currently, CPC is used in liquid form at $75 \mathrm{mg} / 5 \mathrm{ml}$ concentration. Since the dilution of CPC is nullified when using powder form, the concentration of CPC was reduced to $50 \%(38 \mathrm{mg} / 5 \mathrm{~mL})$. All the aliquots were incubated at ambient conditions for 7 days. On day 7 , the aliquots were processed for FDA-EB staining $[4,5]$ and viable count was set up.

2.4. HPLC. Mycolic acids were extraction was carried out as per CDC protocol [6]. Six clinical isolates and one standard strain (H37Rv) were selected and subcultured on LJ medium to get fresh log phase growth. Clinical isolates of $M$. tuberculosis used for mycolic acid analysis were different from those used in viable count. Two loopfuls of the fresh culture was suspended in two milliliters of distilled water, vortexed, and aliquoted into two equal parts. The first aliquot was processed for mycolic acid extraction. The second aliquot was mixed with $7.6 \mathrm{mg}$ of CPC and kept at ambient conditions. On the 7th day, samples were washed with distilled water and processed for HPLC analysis as per the procedure described by Viader-Salvadó et al. [7].

2.5. HPLC Analysis. Chromatography was performed using HPLC system (Agilent 1100 series, Agilent technology Ltd., Germany) with UV detector set at $254 \mathrm{~nm}$ to $350 \mathrm{~nm}$ and data capture unit controlled by software inbuilt in the Agilent
HPLC instrument. Mycolic acid was separated using reverse phase C18 column $(150 \mathrm{~mm} \times 4.6 \mathrm{~mm})$ packed with 5 micron particles (Purospher, Merck Ltd). A solvent gradient system described by Hagen and Thompson was followed with minor modification [8]. After injection, the initial solvent mixture (methanol: methylene chloride-70:30) was maintained for one minute at a constant flow rate of $2.0 \mathrm{~mL} / \mathrm{minute}$. Over the next 15 minutes the solvent composition was changed to 45 : 55 with linear increase. During the next one minute the solvent composition was reverted back to initial composition and the solvent mixture was held to equilibrate the column for 5 minutes. The total elution time was 20 minutes. Mycolic acid elutes between 10 and 13 minutes of run time and the chromatograms were visually compared.

2.6. Pattern Recognition. The chromatographic patterns of $M$. tuberculosis test isolates were visually matched with that of the chromatograms provided in the CDC manual. Reagent blank was treated as negative control to observe any sample contamination. Area under the peaks/cluster was used to calculate the effect of CPC on mycolic acid. Retention time is used to identify and validate the peaks of interest.

\section{Results}

3.1. FDA/EB and VC. FDA/EB consistently stained 70-90\% of $\log$ phase cells as green (Figure 1(a)) and the remaining cells as red-orange. After treating the cells with CPC, 65$70 \%$ of the cells stained red-orange (Figure $1(\mathrm{~b})$ ). These results indicated that FDA entered the mycobacterial cells and hydrolyzed to fluorescein which accumulates intracellularly. These results also indicate that EB could penetrate the lipoid cell wall of the mycobacteria after cell membrane disruption by CPC treatment and intercalate with DNA. The stain also has an ability to penetrate clumps and stain the cells within the clumps. The metabolically active cells ranged from 60 to $90 \%$ on day zero in FDA-EB stain method. Proportion of metabolically active cells on day 7 reduced to 14 to $30 \%$ in controls and 7.5 to $40 \%$ in cells treated with CPC.

The mean log VC on day zero was 6.15. On day 7, the mean log VC in CPC untreated cells and in L-CPC and P-CPC treated cells was 5.92, 5.95, and 6.05, respectively (Table 1). The counts were comparable to zero-day controls.

3.2. HPLC. The representative chromatogram of mycolic acid pattern for the standard strain (H37Rv) obtained from a control before and after CPC treatment was shown as Figures 2(a) and 2(b). Figures 3(a) and 3(b) showed representative chromatograms of mycolic acid obtained from clinical isolates before and after the addition of CPC. Mycolic acid content was reduced in $M$. tuberculosis when treated with CPC (Table 2). Synthesis of mycolic acids in mycobacteria was reduced when exposed to CPC due to the decreased metabolic activity of the organisms.

\section{Discussion}

The FDA-EB staining method is an alternative method of measuring mycobacterial viability. The feasibility of FDA-EB 
TABLE 1: Viable counts and proportion of metabolically active cells in control and CPC exposed cells of M. tuberculosis.

\begin{tabular}{|c|c|c|c|c|c|c|c|c|}
\hline \multirow{3}{*}{ Strains } & \multirow{2}{*}{\multicolumn{2}{|c|}{$\begin{array}{c}0 \text { day } \\
\text { Control }\end{array}$}} & \multicolumn{6}{|c|}{ Day 7} \\
\hline & & & \multicolumn{2}{|c|}{ Control } & \multicolumn{2}{|c|}{ L-CPC } & \multicolumn{2}{|c|}{$\mathrm{P}-\mathrm{CPC}$} \\
\hline & $\mathrm{VC}$ & FDA $^{*}$ & $\mathrm{VC}$ & FDA $^{*}$ & VC & FDA $^{*}$ & VC & $\mathrm{FDA}^{*}$ \\
\hline Standard strain $\left(\mathrm{H}_{37} \mathrm{RV}\right)$ & 6.27 & $90 \%$ & 6.00 & $25 \%$ & 6.17 & $22 \%$ & 6.25 & $22 \%$ \\
\hline Clinical isolate 1 & 6.25 & $80 \%$ & 6.14 & $17 \%$ & 5.90 & $25 \%$ & 6.14 & $17 \%$ \\
\hline Clinical isolate 2 & 5.84 & $80 \%$ & 5.69 & $14 \%$ & 5.84 & $7.5 \%$ & 6.77 & $11 \%$ \\
\hline Clinical isolate 3 & 6.20 & $70 \%$ & 5.77 & $30 \%$ & 5.90 & $20 \%$ & 5.90 & $40 \%$ \\
\hline Clinical isolate 4 & 6.07 & $60 \%$ & 5.84 & $16 \%$ & 5.84 & $20 \%$ & 6.04 & $17 \%$ \\
\hline Mean value $\left(\log _{10}\right)$ & \multicolumn{2}{|c|}{6.15} & \multicolumn{2}{|c|}{5.92} & \multicolumn{2}{|c|}{5.95} & \multicolumn{2}{|c|}{6.05} \\
\hline
\end{tabular}

FDA $^{*}$ expressed as percentage of green live cells present in the culture suspension.

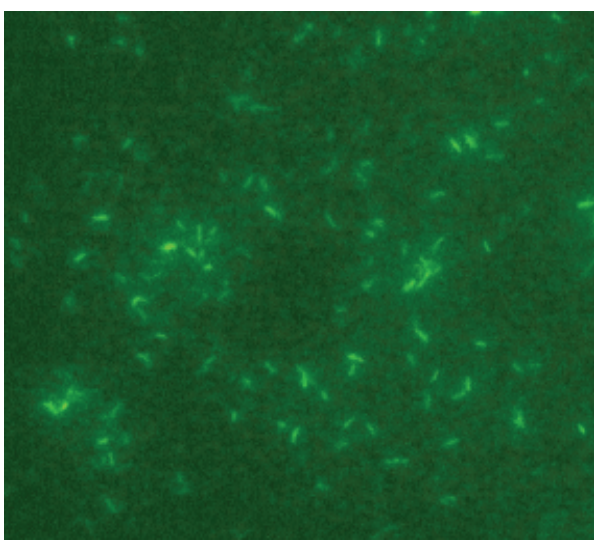

Live cells (green) in 450x were seen

(a) Live cells (Green)

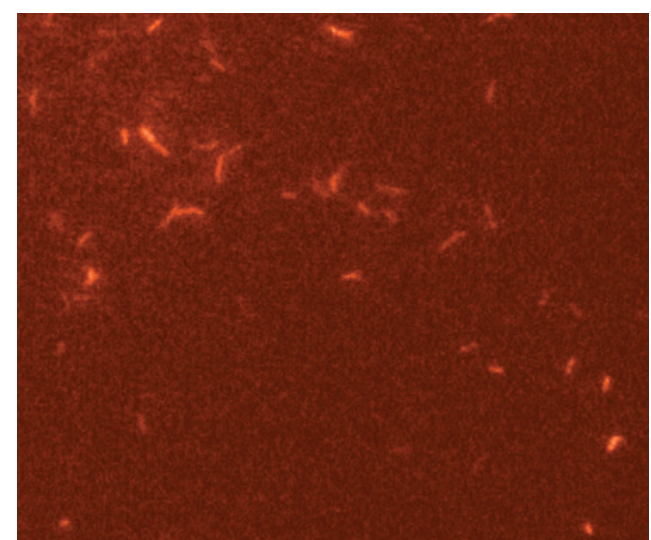

Dead cells (red) in 450x were seen.

(b) Dead cells (Red)

FigURE 1: FDA-EB staining using Fluorescence microscopy.

TABLE 2: Mycolic acid index (MAI) values of clinical isolates.

\begin{tabular}{lcc}
\hline Lab. no. & MAI without CPC & MAI with CPC \\
\hline Standard $\mathrm{H}_{37} \mathrm{Rv}$ & 816.81 & 315.005 \\
Isolate 1 & 3283.01 & 835.877 \\
Isolate 2 & 2945.36 & 1141.29 \\
Isolate 3 & 1378.99 & 835.82 \\
Isolate 4 & 1355.44 & 755.56 \\
Isolate 5 & 580.531 & 294.499 \\
Isolate 6 & 702.76 & 452.895 \\
\hline
\end{tabular}

Minimum onefold decrease was observed in the CPC treated samples.

staining method for determining the viability of mycobacterial cells was investigated [5, 9-13]. The principle of FDA-EB staining method is that mycobacteria hydrolyze fluorescein diacetate to free fluorescein through nonspecific cellular esterase enzyme. Accumulation of fluorescein in metabolically active mycobacterial cells can be detected as green colored cells. Jarnagin and Luchsinger [9], while studying the viability of $M$. tuberculosis, observed a significant decrease in percentage of live bacteria stained as green upon increased periods of chemotherapy. Clinical strains included in this study were isolated sputum specimens from treatment naïve and follow-up patients. Though the isolates were taken from fresh log phase cultures, paucibacillary load in follow-up specimens is one of the factors for varying percentage of viability. This could also be one of the reasons for variation in percentage of viability in FDA-EB staining.

M. tuberculosis when not exposed to CPC possesses acetyl esterase and the cells are green in colour. When it was exposed to CPC, larger proportions were red in colour. These results showed that the cells become metabolically inactive. However, the metabolically inactive organisms grew well in LJ after removing the CPC from the culture. Results of viable count showed a minimal reduction after exposure to CPC. The viability of $M$. tuberculosis is reduced after 72 hours under ambient conditions [14]. The variation in one clinical isolate, number 3 , may be due to the above reason. It is always possible that each clinical isolate behaves differently and it is unexpected. There could also be possibly technical error of using higher inoculums.

Mycolic acids are high molecular-weight, $\lambda$-alkyl- $\beta$ hydroxy fatty acids containing 70 to 90 carbon atoms. Paramasivan et al. [14] used HPLC analysis of mycolic acids to determine the sensitive and resistant strains after treating the cells with the antituberculosis drugs. Numbers of the peaks depicting different mycolic acids were same in CPC treated and untreated samples, but the area was reduced in CPC 


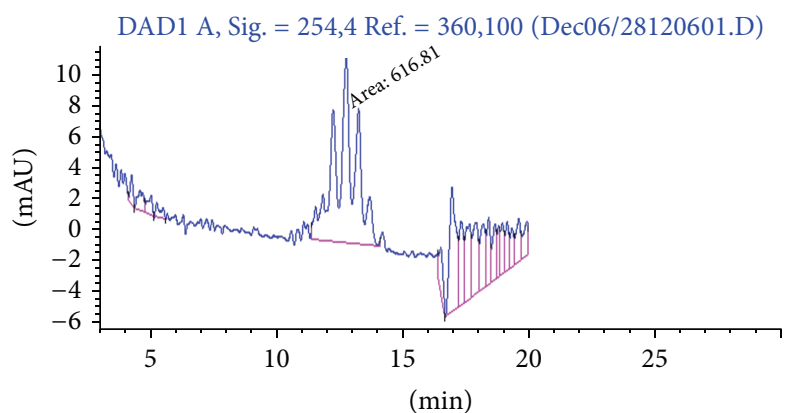

(a) Std strains of H37RV without CPC

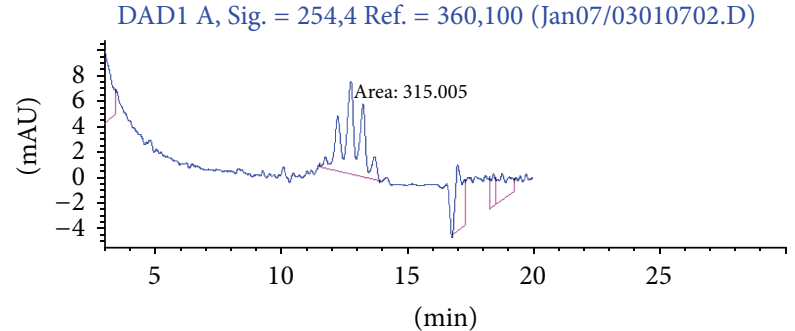

Inference: reduction of MAI on CPC treated cultures was seen

(b) Std strains of H37RV with CPC

FIGURE 2: Determination of mycolic acid content of $M$. tuberculosis after exposure to P-CPC (H37RV).

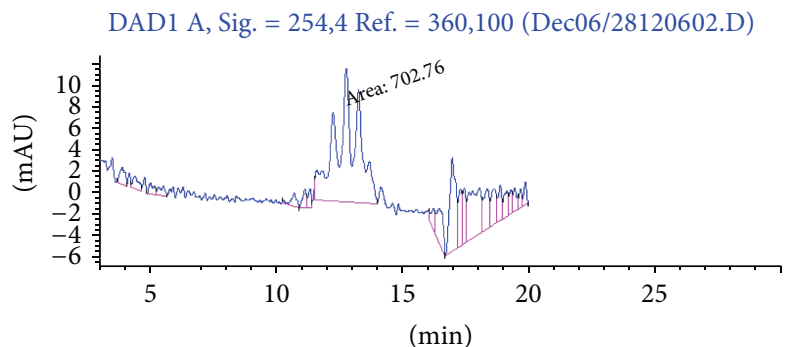

(a) Clinical isolate 6 without CPC

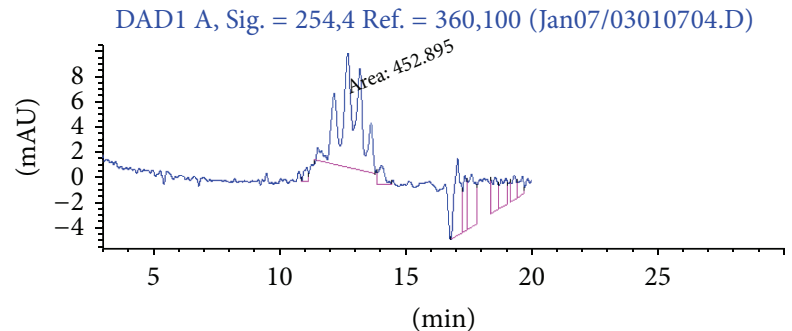

Inference: reduction of MAI on CPC treated cultures was seen

(b) Clinical isolate 6 with CPC

Figure 3: Determination of mycolic acid content of $M$. tuberculosis after exposure to P-CPC (clinical isolate).

treated sample. The CPC reacts with the cell wall of the bacilli and cause damage. Mycolic acid content was shown to be reduced in HPLC analysis. This may be the reason for the reduction of AFB positives in smears stained by AP method, but viable count was not affected. The cell wall damage did not affect the growth and the damaged bacilli grew well on LJ medium. The difference in mycolic acid level between zero-day control and day 7-CPC treated strains indicates that there is decreased production of cell wall components. The cells though in live state do not multiply. CPC treated cells have pleomorphic morphology devoid of cell wall, which is depicted as reduction in mycolic acid index. These cells are however metabolically active as they regain their cell wall and grow when the pressure of CPC is removed. Thus all the above factors indicate that CPC may potentially act on the cell wall and degrade them. This was supported by the electron microscopy images that indicate CPC cells devoid of their outer cove, that is, cell wall (not shown in this paper).

The clinical strains are isolated from both treatment naive as well follow up patients. Hence, there could be difference in the load of bacilli among them which could be depicted in the mycolic acid index. Moreover, M. tuberculosis cells tend to vary in their morphology before and after exposure to Anti tuberculosis drugs. We have observed bacilli with reduction in their size especially after drug treatment. Hence, the amount of mycolic acid will also be less in these cells which could have been the cause for the decreased MAI before CPC treatment in these isolates. This could be one of the reasons for the low MAI even without treatment in two isolates (isolates nos. 5\&6).

HPLC work was performed with P-CPC. Addition of bicarbonate to neutralize the alkaline conditions during extraction procedures reacted with L-CPC. The dark brown color from the reaction posed a hindrance during UV measurements in HPLC. The retention time of CPC and mycolic acid of M. tuberculosis was similar. Moreover, the elution of mycolic acid also became very difficult when using $\mathrm{L}-\mathrm{CPC}$ rather that $\mathrm{P}-\mathrm{CPC}$.

\section{Conclusion}

The cells are metabolically inactive during storage with CPC but these cells grew well on LJ medium after removal of CPC. Viability of $M$. tuberculosis was maintained in CPC with minimal reduction. Mycolic acid content was also reduced when $M$. tuberculosis was treated with CPC for 7 days. This provides yet another evidence for the damage of cell wall of $M$. tuberculosis during CPC treatment.

\section{Conflict of Interests}

The authors declare that there is no conflict of interests regarding the publication of this paper. 


\section{Acknowledgments}

The authors gratefully acknowledge the staff of Department of Bacteriology, NIRT, for their help in laboratory experiments and Indian Council for Medical Research (ICMR), Department of Health Research, New Delhi, for infrastructure and financial support.

\section{References}

[1] World Health Organization, Global Tuberculosis Control: Surveillance, Planning and Financing, WHO, Geneva, Switzerland, 2006.

[2] World Health Organization, Stop TB Partnership, The Stop TB Strategy: Building on and enhancing DOTS to meet the TB-related Millennium Development Goals, WHO, Geneva, Switzerland, 2006.

[3] World Health Organization, The Global Plan to Stop TB, 20062015. Actions for life-towards a world free of tuberculosis, WHO, Geneva, Switzerland, 2006.

[4] S. Islam, F. Rahman, S. K. Munshi, J. Ahmed, S. M. M. Kamal, and R. Noor, "Use of fluorescein diacetate (FDA) staining to detect viable Mycobacterium tuberculosis," Bangladesh Journal of Medical Science, vol. 11, no. 4, 2012.

[5] S. Harada and N. Numata, "Application of FDA/EB staining for the detection of viable or non-viable mycobacteria in clinical specimens," Kekkaku, vol. 67, no. 2, pp. 113-117, 1992.

[6] W. R. Butler and L. S. Guthertz, "Mycolic acid analysis by high-performance liquid chromatography for identification of mycobacterium species," Clinical Microbiology Reviews, vol. 14, no. 4, pp. 704-726, 2001.

[7] J. M. Viader-Salvadó, E. Garza-González, R. Valdez-Leal, M. de los Angeles del Bosque-Moncayo, R. Tijerina-Menchaca, and M. Guerrero-Olazaran, "Mycolic acid index susceptibility method for Mycobacterium tuberculosis," Journal of Clinical Microbiology, vol. 39, no. 7, pp. 2642-2645, 2001.

[8] S. R. Hagen and J. D. Thompson, "Analysis of mycolic acids by high-performance liquid chromatography and fluorimetric detection. Implications for the identification of mycobacteria in clinical samples," Journal of Chromatography A, vol. 692, no. 1-2, pp. 167-172, 1995.

[9] J. L. Jarnagin and D. W. Luchsinger, "The use of fluorescein diacetate and ethidium bromide as a stain for evaluating viability of mycobacteria," Stain Technology, vol. 55, no. 4, pp. 253-258, 1980.

[10] J. T. Kvach and J. R. Veras, "A fluorescent staining procedure for determining the viability of mycobacterial cells," International Journal of Leprosy, vol. 50, no. 2, pp. 183-192, 1982.

[11] J. T. Kvach, G. Munguia, and S. H. Strand, "Staining tissuederived Mycobacterium leprae with fluorescein diacetate and ethidium bromide," International Journal of Leprosy, vol. 52, no. 2, pp. 176-182, 1984.

[12] V. M. Katoch, K. Katoch, U. Ramanathan et al., "Effect of chemotherapy on viability of Mycobacterium leprae as determined by ATP content, morphological index and FDA-EB fluorescent staining," International Journal of Leprosy, vol. 57, no. 3, pp. 615-621, 1989.

[13] E. Garza-Ggonzález, M. Guerrero-Olazarán, R. TijerinaMenchaca, and J. M. Viader-Salvadó, "Determination of drug susceptibility of Mycobacterium tuberculosis through mycolic acid analysis," Journal of Clinical Microbiology, vol. 35, no. 5, pp. 1287-1289, 1997.
[14] C. N. Paramasivan, A. S. L. Narayana, R. Prabhakar, M. S. Rajagopal, P. R. Somasundaram, and S. P. Tripathy, "Effect of storage of sputum specimens at room temperature on smear and culture results," Tubercle, vol. 64, no. 2, pp. 119-124, 1983. 

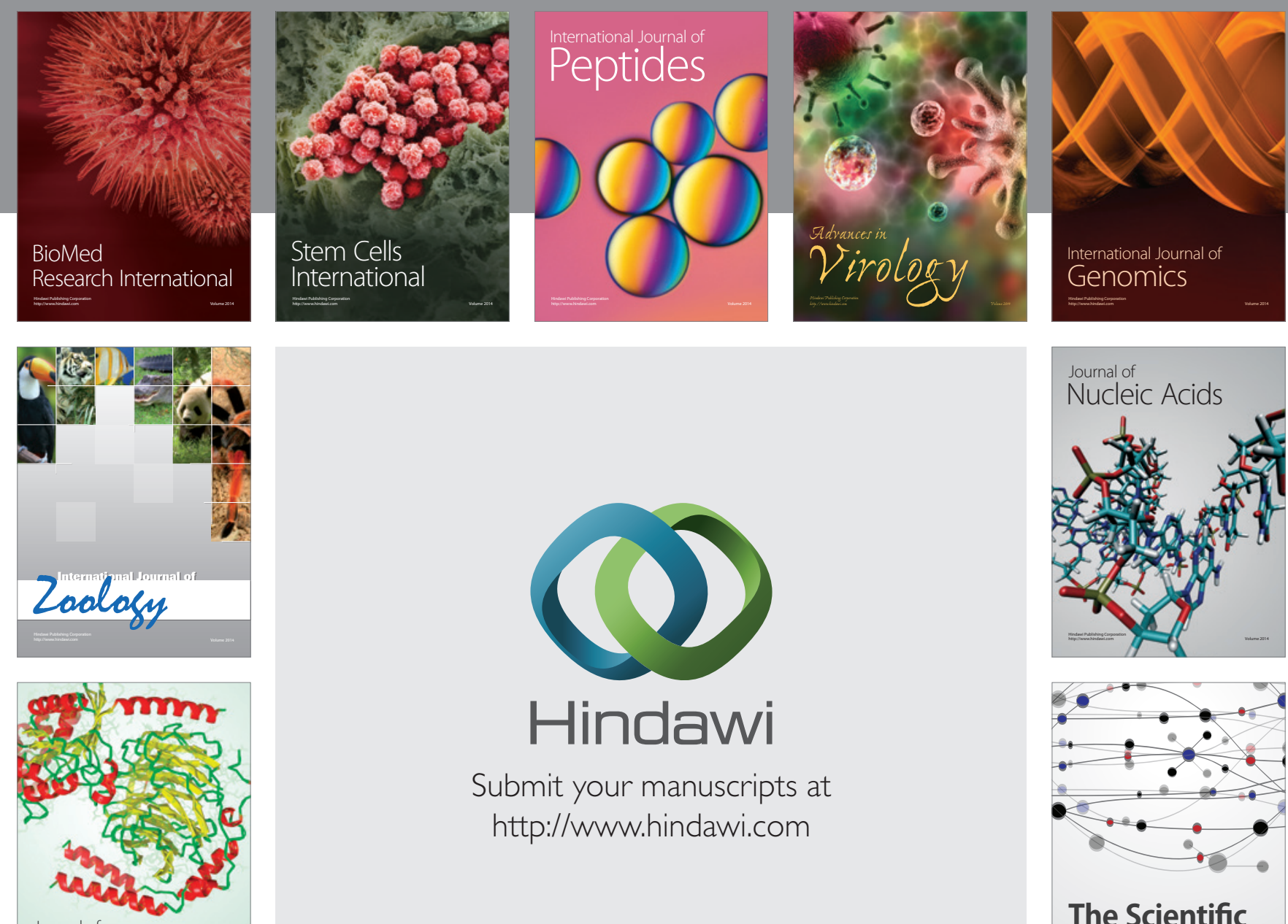

Submit your manuscripts at

http://www.hindawi.com

Journal of
Signal Transduction
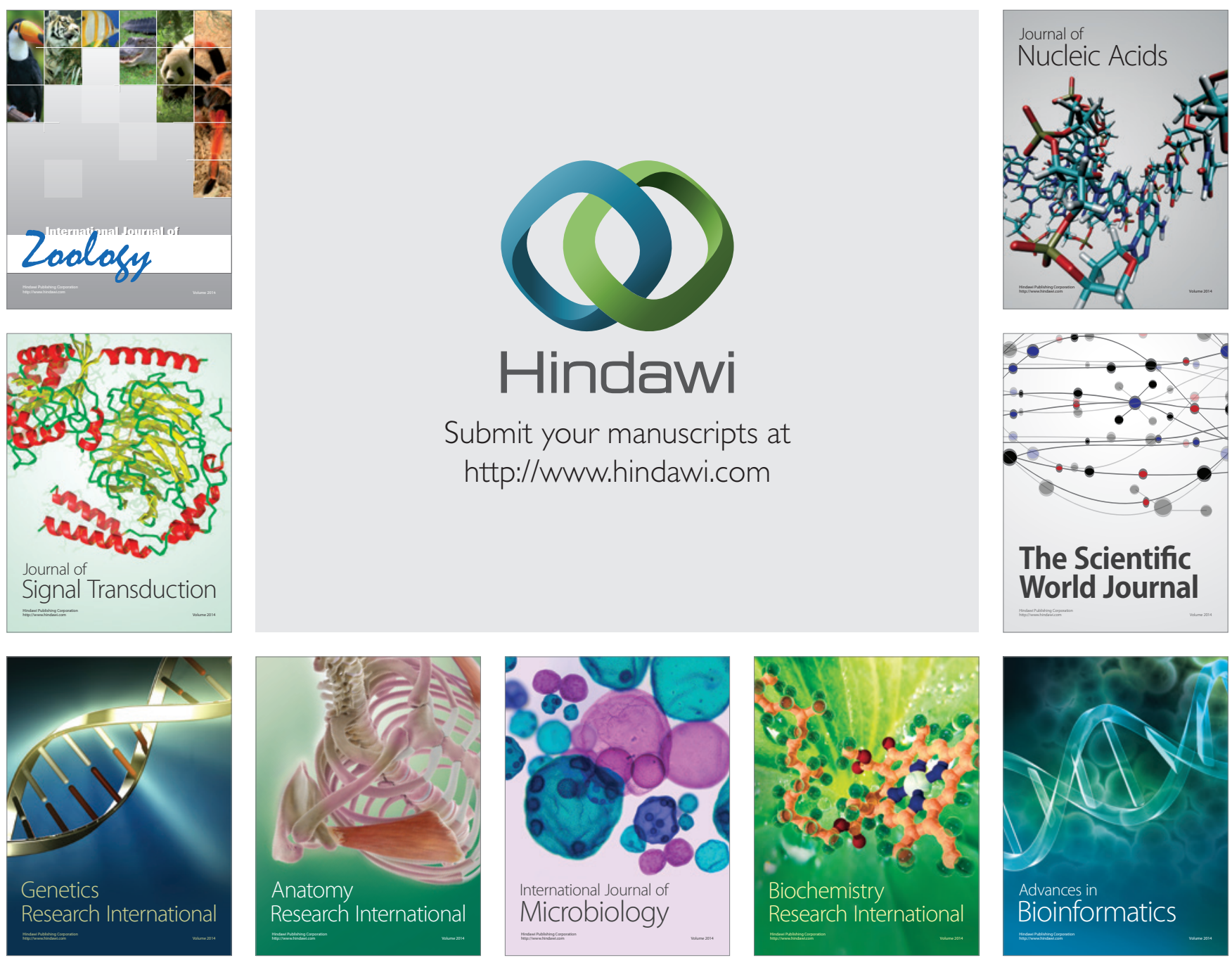

The Scientific World Journal
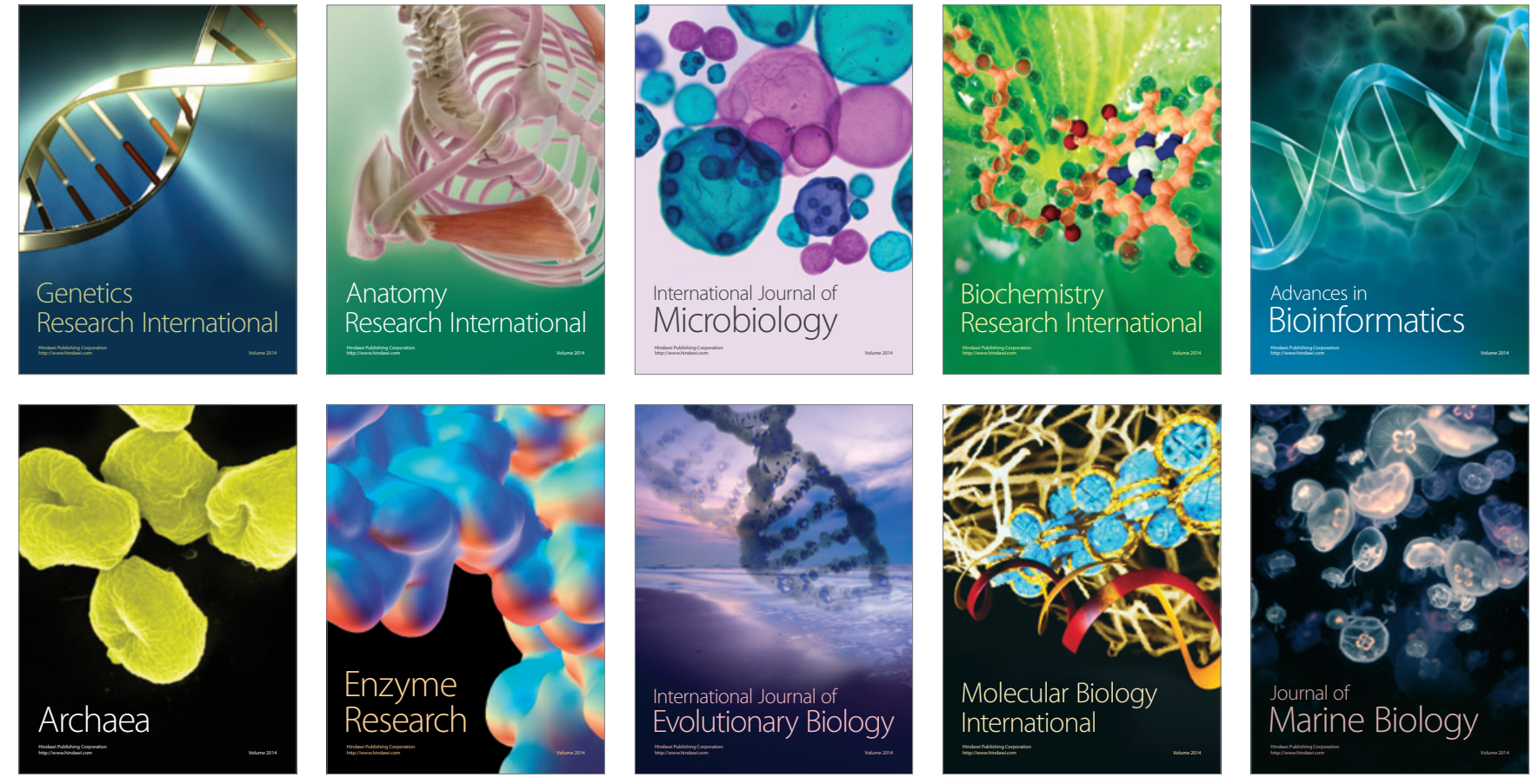